\title{
Identification of miRNA-mRNA network in NPM1 mutated acute myeloid leukemia
}

Syed Khizer Hasan ${ }^{1}$, Nikhil Gadewal ${ }^{1}$, Swapnil Aher ${ }^{1}$, Anagha Gardane ${ }^{1}$, Rohit Kumar ${ }^{1}$, Ashok Varma ${ }^{1}$ and Navin Khattry ${ }^{2}$ ${ }^{1}$ ACTREC, Tata Memorial Centre, Navi-Mumbai, India. ${ }^{2}$ Adult Hematolymphoid Disease Management Group, Department of Medical Oncology, Tata Memorial Centre, Mumbai, India

\section{RESULTS}

- Quantitative expression of NPM1 mutation A by RQ-PCR (Left panel) and Western blot (Right panel) - AML with

- Mutant NPM1 AML has unique microRNA and their target gene (mRNA)

- Dynamic regulation of miRNA-mRNA has been reported to influence prognostic outcome.

- NPM1 mutation induces chemo-sensitivity in leukemic cells but the underlying cause for the better survival of NPM1 mutated patients is still not clear.

\section{AIM OF THE STUDY}

- To evaluate the pairwise correlations of differential expression between miRNA and $m R N A$ and if the strength of differential (negative or positive) regulation of a miRNA on its target gene can be modulated by NPM1 mutation in AML. MATERIALS AND METHODS

\section{ranscriptomics analysis from Genomic Data Commons (GDC)}

- In silico analyses (Schematic representation shown below)

$$
\begin{aligned}
& \text { In silico data } \\
& \text { patients data from }
\end{aligned}
$$

GDC portal ( $(n=183)$

GDC portal (r

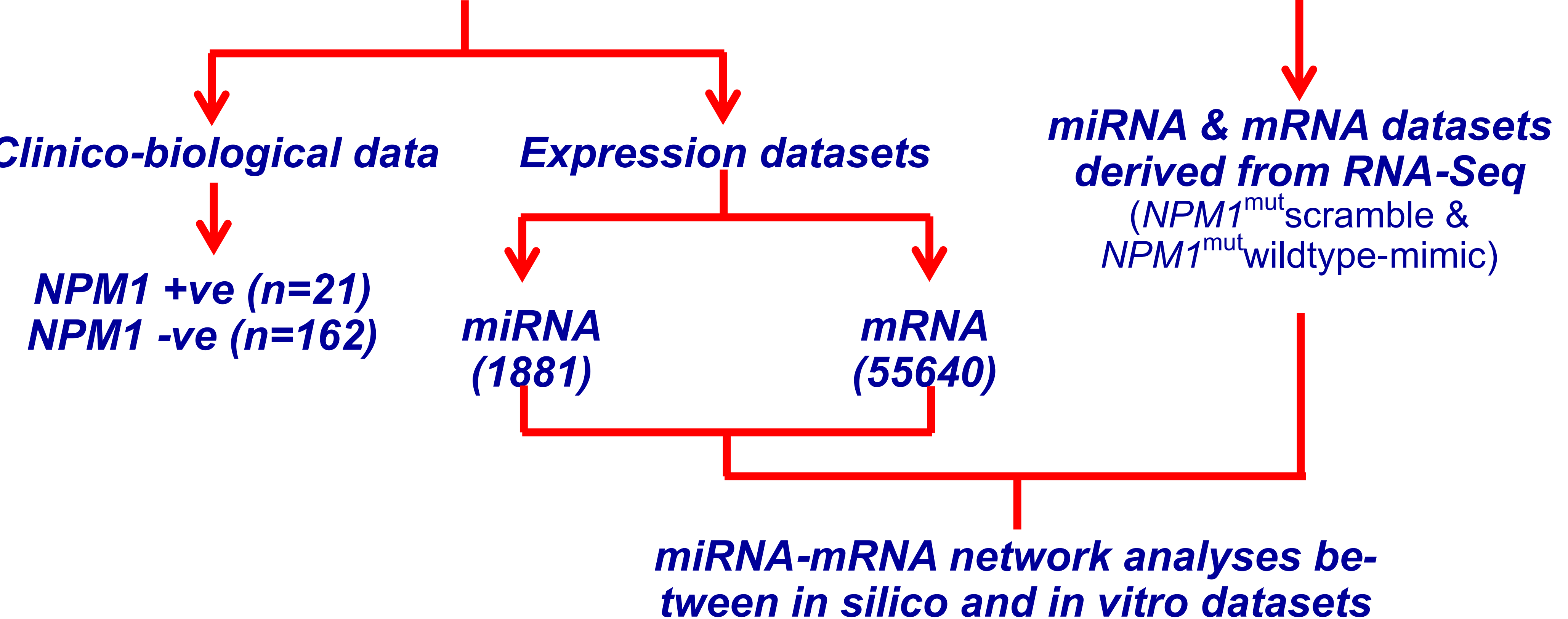

High throughput transcriptional profiling (RNA sequencing)- In-vitro analyses

- Knockdown of NPM1 mutation in OCI/AML3 cells by NPM1 mutation specific

- Evaluation of NPM1 mutation knockdown efficiency by real time quantitative PCR (RQ-PCR) and Western blotting

High throughput RNA sequencing using NPM1 ${ }^{\text {mut }}$ scramble and NPM1 ${ }^{\mathrm{m}}$ wildtype-mimic and data analyses.

Patient samples, cDNA synthesis and RQ-PCR

- To validate the impact of $S M C 1 A$ expression on survival between $N P M 1^{\text {mut }}$ and $N P M 1^{\text {wt }}$ patients in an independent cohort of AML patients, bone marrow samples from 67 AML patients at the time of diagnosis were collected. All patients provided written informed consent. The SYBR green based RQ-PCR assays for SMC1A was carried out using in house designed primers and standard protocols.

\section{STATISTICAL ANALYSES}

- Overall Survival (OS): From the date of start of induction therapy to date of last - follow up or death. Results were analyzed by Kaplan-Meier method and compared using log-rank test. Statistical analysis was done using IBM SPSS v21.

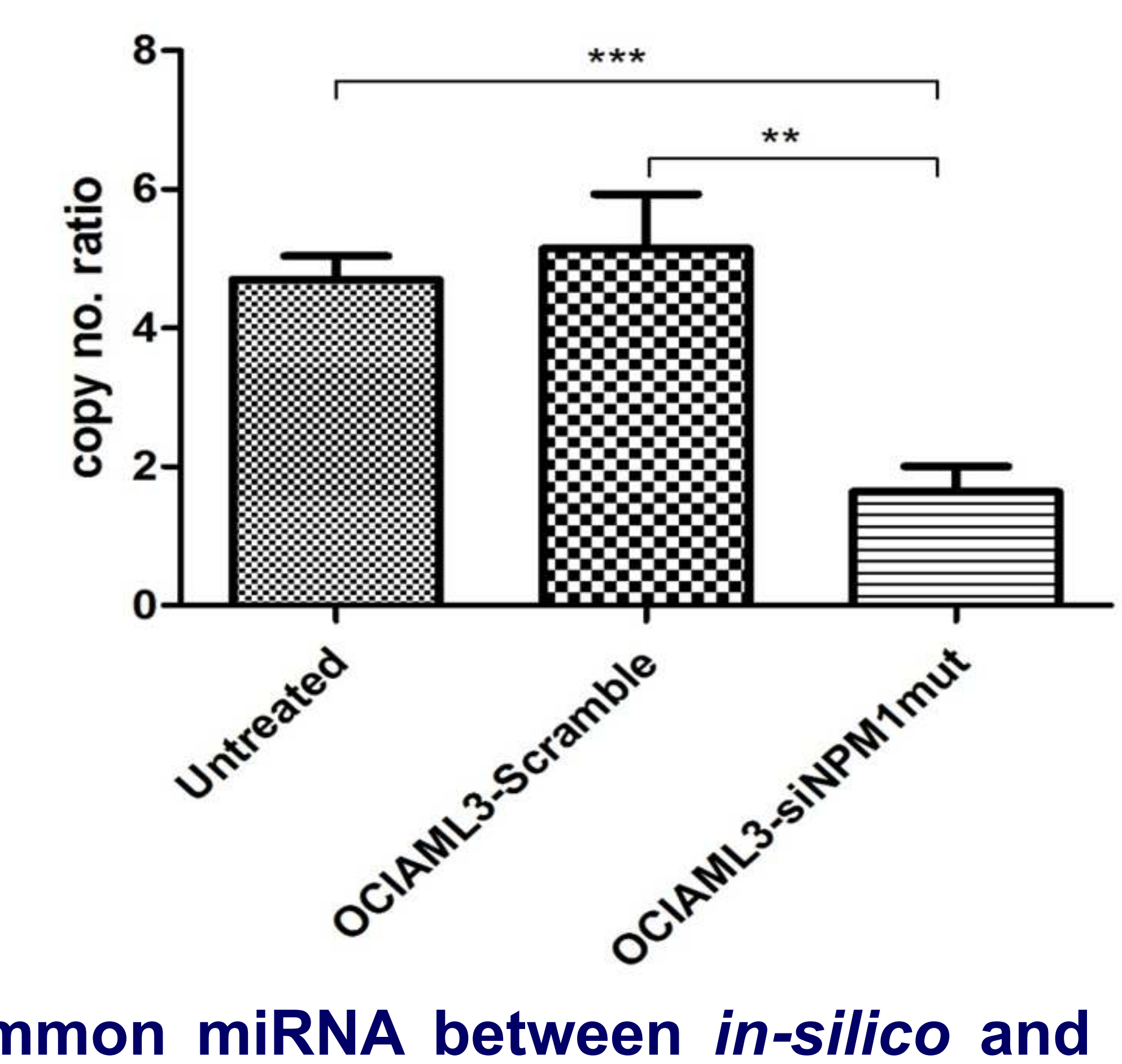

- Common miRNA between in-silico and in-vitro datasets of AML with NPM1 mutation compared to NPM1 wildtype AML

\begin{tabular}{|c|c|c|c|c|}
\hline \multirow{2}{*}{ miRNA } & \multicolumn{3}{|c|}{ In-silico dataset } & \multirow{2}{*}{$\begin{array}{c}\text { In-vitro dataset } \\
\text { Log2 FC }\end{array}$} \\
\hline & Log2 FC & $p$-value & $F D R$ & \\
\hline hsa-mir-363 & 1.9336 & 0.0002 & 0.0105 & -2.4921 \\
\hline hsa-mir-1291 & -1.3753 & 0.0004 & 0.0176 & 3.9508 \\
\hline hsa-mir-607 & 2.3239 & 0.0014 & 0.0405 & -4.1551 \\
\hline hsa-mir-7976 & -1.1299 & 0.0020 & 0.0541 & -2.0771 \\
\hline hsa-mir-382 & -1.9266 & 0.0027 & 0.0623 & 2.4148 \\
\hline hsa-mir-3912 & 1.0942 & 0.0031 & 0.0677 & 2.0188 \\
\hline hsa-mir-6718 & -1.2294 & 0.0045 & 0.0841 & -2.2291 \\
\hline hsa-mir-3911 & 2.7209 & 0.0047 & 0.0851 & -2.9072 \\
\hline hsa-mir-5087 & 1.8014 & 0.0081 & 0.1154 & 2.4148 \\
\hline hsa-mir-4786 & 1.0669 & 0.0088 & 0.1203 & -3.3666 \\
\hline hsa-mir-450a-1 & 1.4821 & 0.0134 & 0.1545 & 2.2221 \\
\hline hsa-mir-215 & 0.9579 & 0.0189 & 0.1765 & 2.8298 \\
\hline hsa-mir-6747 & 1.2568 & 0.0339 & 0.2443 & -2.4921 \\
\hline hsa-mir-449a & 1.9596 & & & \\
\hline
\end{tabular}

- miRNA-mRNA interactive pairs derived from in-silico and in-vitro analyses of

\begin{tabular}{|c|c|c|c|c|c|}
\hline miRNA & Mature miRNA & $\begin{array}{c}\text { Genes } \\
\text { (mRNA) }\end{array}$ & $\begin{array}{l}\text { Log2 FC } \\
\text { (in-silico } \\
\text { dataset) }\end{array}$ & $\begin{array}{c}\text { p value } \\
\text { (in-silico da- } \\
\text { taset) }\end{array}$ & $\begin{array}{l}\text { Log2 FC } \\
\text { (in-vitro da- } \\
\text { taset) }\end{array}$ \\
\hline hsa-miR-363 & hsa-miR-363-3p & NRAS & 0.0614 & 0.7581 & 0.3042 \\
\hline \multirow{3}{*}{ hsa-mir-607 } & \multirow{3}{*}{ hsa-mir-607 } & NPM1 & 0.2240 & 0.1686 & 0.4103 \\
\hline & & PTEN & -0.3414 & 0.0846 & 0.1482 \\
\hline & & SRSF2 & -0.0263 & 0.8232 & 0.0504 \\
\hline \multirow{3}{*}{ hsa-mir-382 } & \multirow{3}{*}{ hsa-miR-382-5p } & PTEN & -0.3414 & 0.0846 & 0.1482 \\
\hline & & SF3B1 & -0.1508 & 0.4729 & 0.0647 \\
\hline & & SMC3 & -0.1740 & 0.3946 & 0.2259 \\
\hline \multirow{6}{*}{ hsa-miR-215 } & hsa-miR-215-3p & SMC1A & -0.4816 & 0.0464 & 0.7043 \\
\hline & \multirow{5}{*}{ hsa-miR-215-5p } & $\mathrm{BCOR}$ & 0.1747 & 0.3525 & 0.5026 \\
\hline & & CDKN2A & 0.6033 & 0.1625 & 0.5748 \\
\hline & & PHF6 & -0.2824 & 0.3182 & 0.5899 \\
\hline & & RAD21 & -0.1296 & 0.4879 & 0.2637 \\
\hline & & WT1 & 0.0509 & 0.9041 & -0.0557 \\
\hline hsa-mir-449a & hsa-mir-449a & $\mathrm{NOTCH} 1$ & -0.0187 & 0.9394 & 0.3419 \\
\hline
\end{tabular}

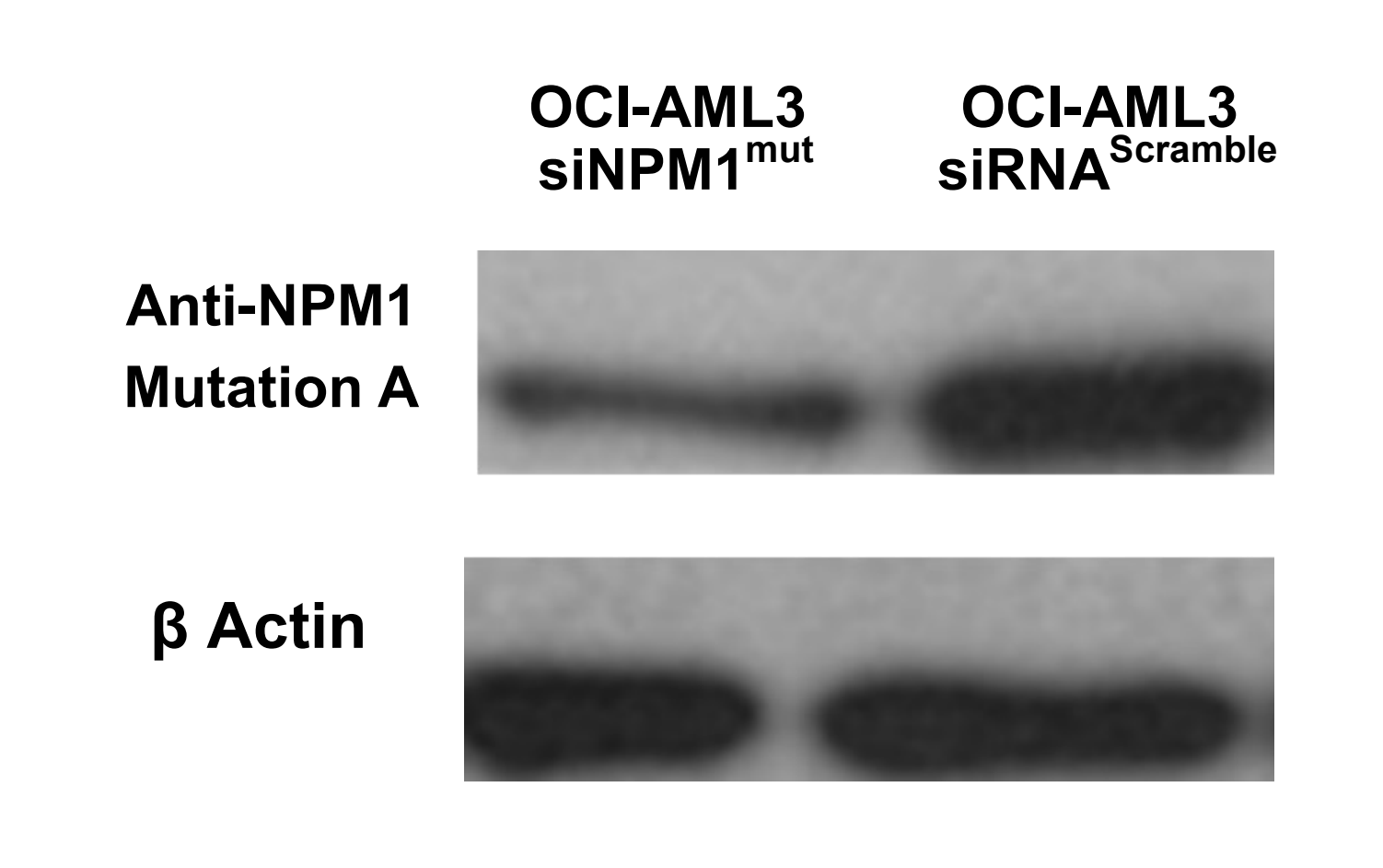
NPM1 mutated AML
- SMC1A median expression* versus survival outcome A) NPM1 non-mutated ( $\mathrm{N}=160$ ); B) NPM1 mutated AML patients ( $\mathrm{N}=20)$
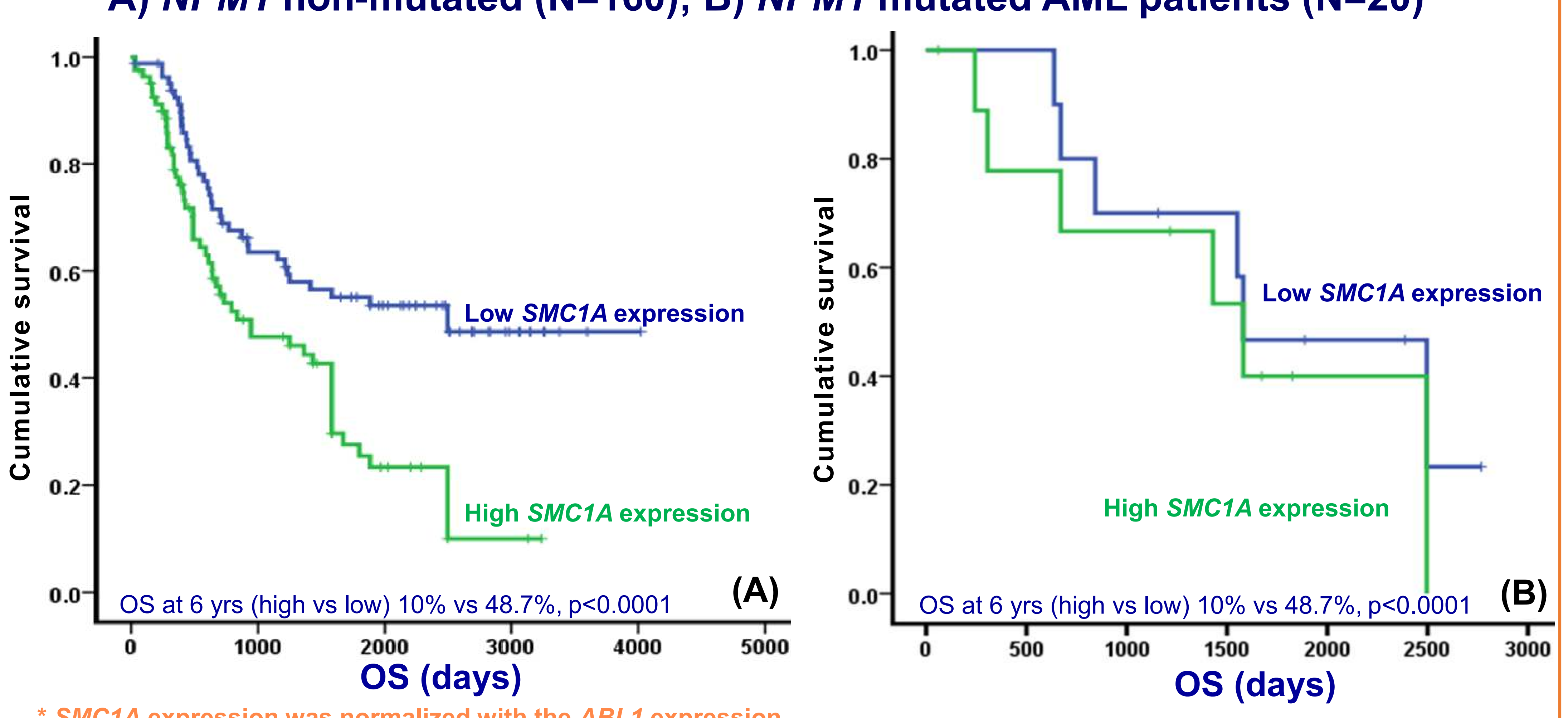

Validation of SMC1A Expression in an

- Seventeen patients were positive for NPM1 type A mutation while $\mathbf{5 0}$ cases were negative for the mutation. The median age of the cohort was 27 years (range 15-58 yrs). Out of 67 patients, three patients died during induction, five were refractory to the treatment while the follow up data was not available for two patients and hence these cases were excluded from os analysis. The OS of 57 patients was $28.5 \%$ at 3 years.

- SMC1A median expression* versus survival outcome in an independent cohort of AML patients

A) NPM1 wild type ( $N=40)$ B) NPM1 mutated patients $(\mathrm{N}=17)$
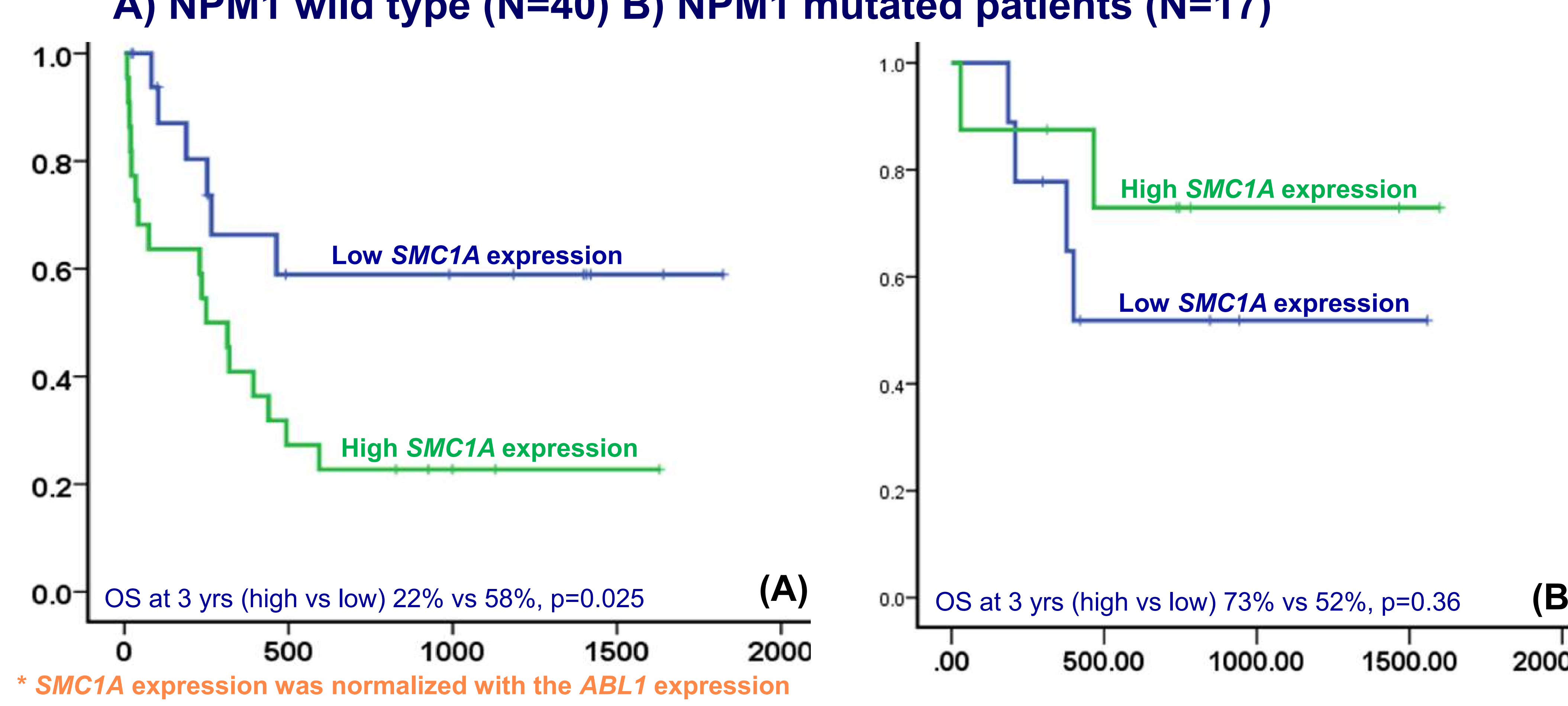

\section{CONCLUSION}

The down-regulation of SMC1A mediated by miR-215 suggesting its role behind the better prognosis of NPM1 mutated AML as compared to AML with wildtype NPM1. Considering the small cohort patients in the present study, the future studies are warranted to confirm these findings in a larger cohort of AML patients.

\section{ACKNOWLEDGEMENTS}

The authors are grateful to Prof. F. Lo-Coco from Rome, Italy, who has provided NPM1 mutation specific antibody and IEC, and Tata Memorial Centre Mumbai for funding. 